\title{
Comprehensive confocal endomicroscopy of the esophagus in vivo
}

Authors

Institutions
Dongkyun Kang' ${ }^{1}$ Simon C. Schlachter ${ }^{1}$, Robert W. Carruth ${ }^{1}$, Minkyu Kim ${ }^{1,2}$, Tao Wu ${ }^{1}$, Nima Tabatabaei ${ }^{1}$, Paulino Vacas-Jacques ${ }^{1}$, Milen Shishkov ${ }^{1}$, Kevin Woods ${ }^{2,3}$, Jenny S. Sauk ${ }^{3}$, John Leung ${ }^{3}$, Norman S. Nishioka ${ }^{3}$, Guillermo J. Tearney ${ }^{1,4,5}$

Institutions are listed at the end of article. submitted 30. March 2014 accepted 18. April 2014

\section{Bibliography}

Dol http://dx.doi.org/

10.1055/s-0034-1377177

Published online: 16.7.2014

Endoscopy International Open

2014; 02: E135-E140

(c) Georg Thieme Verlag KG

Stuttgart · New York

E-ISSN 2196-9736

\section{Corresponding author}

Guillermo J. Tearney, MD, PhD

Massachusetts General Hospital

- Wellman Center for

Photomedicine

40 Blossom St. BHX

604 Boston, MA 02114

United States

Fax: 6177264103

gtearney@partners.org
Background and study aims: Biopsy sampling error can be a problem for the diagnosis of certain gastrointestinal tract diseases. Spectrally-encoded confocal microscopy (SECM) is a high-speed reflectance confocal microscopy technology that has the potential to overcome sampling error by imaging large regions of gastrointestinal tract tissues. The aim of this study was to test a recently developed SECM endoscopic probe for comprehensively imaging large segments of the esophagus at the microscopic level in vivo.

Methods: Topical acetic acid was endoscopically applied to the esophagus of a normal living swine. The $7 \mathrm{~mm}$ diameter SECM endoscopic probe was transorally introduced into the esophagus over a wire. Optics within the SECM probe were helically scanned over a $5 \mathrm{~cm}$ length of the esophagus. Confocal microscopy data was displayed and stored in real time.

\section{Introduction}

$\nabla$

Standard of care endoscopic biopsy suffers from sampling error, which can limit the diagnosis of many esophageal diseases. Confocal laser endomicroscopy (CLE) is a recently introduced imaging technique where a microscopy probe is inserted into the gastrointestinal tract to obtain microscopic images of tissue without biopsy excision [1 -5]. While CLE has the potential to improve tissue sampling [6], the speeds and modes of operation of CLE devices prohibit automatic and comprehensive acquisition of microscopic image data over large segments of luminal gastrointestinal organs.

Spectrally-encoded confocal microscopy (SECM) is a new reflectance CLE technology that uses a diffraction grating and a wavelength-swept laser to image tissues at very high speeds (>10 $\times$ video rate) [7]. Because of its high-speed capabilities, SECM has a potential to image the entire distal esophagus in an acceptable procedural time. Dif-
Results: Very large confocal microscopy images (length $=5 \mathrm{~cm}$; circumference $=2.2 \mathrm{~cm}$ ) of swine esophagus from three imaging depths, spanning a total area of $33 \mathrm{~cm}^{2}$, were obtained in about 2 minutes. SECM images enabled the visualization of cellular morphology of the swine esophagus, including stratified squamous cell nuclei, basal cells, and collagen within the lamina propria.

Conclusions: The results from this study suggest that the SECM technology can rapidly provide large, contiguous confocal microscopy images of the esophagus in vivo. When applied to human subjects, the unique comprehensive, microscopic imaging capabilities of this technology may be utilized for improving the screening and surveillance of various esophageal diseases.

ferences between CLE and SECM are summarized in Table 1. Previously, SECM, implemented using a bench top microscope, has been shown to be capable of visualizing key histomorphologic features associated with various esophageal diseases ex vivo $[8,9]$. Recently, we have developed a miniature endoscopic SECM probe that contains high-resolution optics [10], can be placed into the gastrointestinal tract endoscopically over a guide wire, and automatically images over large swatches of gastrointestinal tissues using a helical scan pattern. The aim of this study was to test this SECM endoscopic probe for imaging large areas of the esophagus in vivo.

\section{Materials and methods \\ $\nabla$}

SECM endoscopic imaging setup

We conducted comprehensive confocal microscopy using an SECM endoscopic imaging setup shown in Fig. 1. Near infrared light from the 
Table 1 Comparison of eCLE (endoscope-based CLE), pCLE (probe-based CLE) and SECM.

\begin{tabular}{|llll} 
& eCLE & PCLE & SECM \\
\hline Image acquisition speed & $0.8 \mathrm{frames} / \mathrm{sec}^{1}$ & $12 \mathrm{frames} / \mathrm{sec}^{2}$ & $100 \mathrm{frames} / \mathrm{sec}$ \\
\hline Contrast mechanism & Fluorescence & Fluorescence & Reflectance \\
\hline Contrast agent & Intravenous fluorescein & Intravenous fluorescein & Topical acetic acid \\
\hline Implementation & Integrated in endoscope & Though an accessary port & Over a guide wire \\
\hline Laser wavelength & $488 \mathrm{~nm}^{1}$ & $488 \mathrm{~nm}^{2}$ & $1300 \mathrm{~nm}$ \\
\hline Resolution & Lateral: $0.7 \mu \mathrm{m}^{1}$ & Lateral: $1 \mu \mathrm{m}^{2}$ & Lateral: $2 \mu \mathrm{m}$ \\
\hline Imaging depth & Axial: $7 \mu \mathrm{m}^{1}$ & Axial: $3 \mu \mathrm{m}^{2}$ & Axial: $17 \mu \mathrm{m}$ \\
\hline Field of view (FOV) & $0-250 \mu \mathrm{m}^{1}$ & $0-50 \mu \mathrm{m}^{3}$ & $86-114 \mu \mathrm{m}^{4}$ \\
\hline Contiguous mosaic size & $500 \times 500 \mu \mathrm{m}^{21}$ & $240 \times 200 \mu \mathrm{m}^{22}$ & $280 \times 1300 \mu \mathrm{m}^{2}$ \\
\hline Large-area imaging method & No published value available & $8 \mathrm{~mm}^{25}$ & $1,100 \mathrm{~mm}^{26}$ \\
\hline
\end{tabular}

${ }^{1}$ These values were taken from Kiesslich et al. [1].

2 These values were taken from Becker et al. [6].

${ }^{3}$ Imaging depth of pCLE can be set as a fixed value between 0 and $50 \mu \mathrm{m}$ [16].

${ }^{4}$ Multiple depth levels are imaged simultaneously over this range.

${ }^{5}$ This value was taken from De Palma et al. [17].

${ }^{6}$ This value is for a 5 -cm-long pullback of the probe.

laser was delivered to the $7 \mathrm{~mm}$ diameter SECM probe inside the esophagus through a 2-m-long optical fiber. The light was then focused inside the tissue by the SECM probe optics. The reflected light from the tissue was collected by the same SECM probe optics and delivered back to the SECM system. The SECM endoscopic probe had a lateral resolution of $2.3 \mu \mathrm{m}$, an axial resolution of $17 \mu \mathrm{m}$, and a field size of $280 \mu \mathrm{m}$. The focal plane was located $100 \mu \mathrm{m}$ below the tissue surface, and was slightly tilted to image multiple depths during a single scan [11]. The line image of the tissue was acquired at the rate of $100 \mathrm{kHz}$, which resulted in the $1024 \times 1024$-pixel frame rate of 100 frames/sec. The SECM endoscopic probe was helically scanned at a rotation rate of $360 \mathrm{rpm}$ and a pullback rate of $0.4 \mathrm{~mm} / \mathrm{sec}$ to automatically obtain large, contiguous confocal microscopy images of the esophagus.

\section{In vivo swine imaging}

SECM imaging of swine esophagus in vivo was conducted according to a study protocol approved by the Massachusetts General Hospital Subcommittee on Research Animal Care (Protocol\# 2011N000115). Imaging was performed in a $45 \mathrm{~kg}$ female Yorkshire swine. After sedation and intubation, a videoendoscope (EPM-3300, Pentax) was advanced transorally to the gastroesophageal junction, and the initial evaluation of the esophagus was conducted. To increase the contrast of squamous nuclei, [12] a low-concentration of acetic acid ( $6 \%$ concentration by volume) was topically applied to the esophagus using a spray catheter. A guidewire was then introduced to the stomach through the auxiliary channel. The videoendoscope was then removed while leaving the guidewire in place. The SECM endoscopic probe was introduced over the guidewire to approximately $5 \mathrm{~cm}$ proximal from the gastroesophageal junction. Once the probe was in place, the optics automatically rotated and pulled back within the transparent imaging tube. Light returned from the esophagus was detected and stitched together to form contiguous confocal microscopy images in 3 planes over a 5 -cm-long esophageal segment. The animal was euthanized immediately following the procedure. At necropsy, histological examination of the esophagus was performed to conduct a comparative analysis.

\section{Results \\ $\nabla$}

\section{Comprehensive confocal image of swine esophagus} in vivo

Contiguous $5 \mathrm{~cm}$ (length) by $2.2 \mathrm{~cm}$ (circumference) reflectance confocal microscopy images were acquired from three depth planes in 2.1 minutes. A large-area confocal image from the middle depth is shown in $\bullet$ Fig. 2 a. The horizontal axis of the image corresponds to the longitudinal direction of the esophagus, and the vertical axis is the circumferential axis. Approximately $22 \mathrm{~cm}^{2}(65 \%)$ of the imaged area contains microscopic tissue

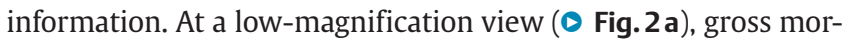
phology of the esophagus can be seen. The epithelium (E) and lamina propria (LP) are visualized, and numerous papillae are seen as circular features with low SECM signal (arrows). Portions of the SECM image $(\sim 35 \%)$ are artifactual; in the top portion of the image, the imaging tube lost contact with the esophagus (NC) and the focal plane of the probe was not inside the tissue. At the bottom of the image, a segment of the image reveals high reflectance at the border between the liquid-air interface (LA). As shown in $-\mathbf{F i g .} \mathbf{2} \mathbf{b}$, the large-area image can be zoomed in to show a small region of interest at a higher magnification. The size of $\bullet$ Fig. 2 b is $2.0 \mathrm{~mm}$ by $1.3 \mathrm{~mm}$, which is similar in size to a lowpower microscopic field used in histologic analysis. At this magnification, cellular features of the tissue can be clearly seen, including numerous nuclei (seen as bright, highly reflecting dots) surrounding two papillae (shown as dark circular regions of low SECM signal marked by arrows). The image can be zoomed in further to produce a view ( Fig. $\mathbf{2 c}$ ) that is similar in size to a highpower field $(40 \times)$. At this high magnification, numerous nuclei (N) can be seen encircling a papilla.

Cellular features were visualized in SECM images at various depths within the epithelium. $\bullet$ Fig. 3 shows high-magnification SECM images at three distinctive locations and en face histologic images of the swine esophagus. Fig. 3 a, a SECM image taken from a superficial plane within the epithelium (box 1 in $\bullet$ Fig. 2 a), shows scattered nuclei (bright dots) similar to that seen in a histologic image of representative, nearby superficial squamous epithelium ( Fig.3d). Fig.3b, a SECM image taken from a deeper region of the epithelium (box 2 in $\bullet$ Fig.2a), demonstrates numerous nuclei (bright dots) with a higher density than - Fig.3a and multiple papillae (dark circular regions) sur- 


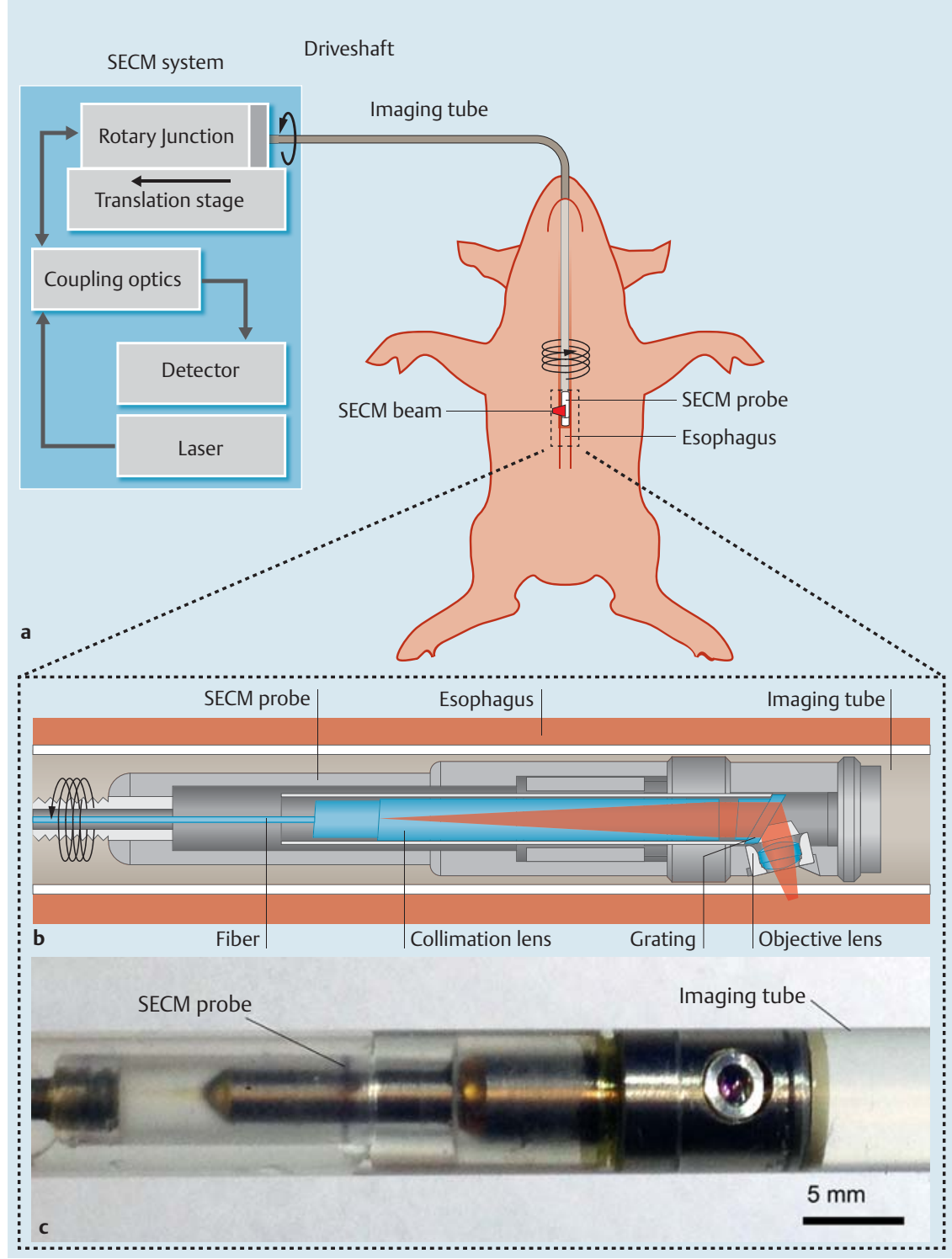

Fig. 1 Schematics and photo of the SECM endoscopic imaging setup. a Overview of the SECM endoscopic imaging setup. An SECM endoscopic probe is inserted into an imaging tube, which is a transparent semi-flexible plastic tube. The imaging tube is introduced to the esophagus transorally. A SECM beam is focused into the esophageal tissue. While SECM images are continuously acquired, the SECM probe is helically scanned by a rotary junction and translation stage to image a large area of the esophagus. b Detailed schematic of the SECM probe optics. In the probe optics, light from the fiber is collimated by a collimation lens and diffracted by a grating. The diffracted light is focused by an objective lens (water immersion; numerical aperture $=0.5$ ) into a $280-\mu \mathrm{m}$-long line. $\mathrm{c}$ Photo of the SECM optical probe inside the imaging tube. The diameter of the probe is $5.9 \mathrm{~mm}$, and the rigid length is $30 \mathrm{~mm}$. The outer diameter of the imaging tube is $7.0 \mathrm{~mm}$.

rounded by the nuclei. Similar cellular features are observed in a representative histologic image of the basal cell layer ( $\bullet$ Fig.3e). - Fig. 3 c, an SECM region from the LP (box 3 in $\bullet$ Fig.2a), enables the visualization of microstructures with high SECM signals that are similar in appearance to collagen seen in a representative histologic image of the LP $(\bullet$ Fig. $\mathbf{3 f})$.

\section{Imaging multiple depths in a single scan}

SECM images from multiple imaging depths were obtained through post-operation image processing of the volumetric data that was acquired in a single helical scan of the probe [11] - Fig. 4 shows SECM and en face histologic images obtained from the same respective transverse locations but at different imaging depths. The imaging depth changes from superficial $(\bullet$ Fig. $4 \mathrm{a}$ and d) to deep ( $\bullet$ Fig.4c and $\mathrm{f}$ ) with a depth interval of $14 \mu \mathrm{m}$ between images. The SECM images were taken from the box 4 in Fig. 2 a. In the SECM images, the papillae (dark circular regions) become bigger as the imaging depth increases from Fig. 4 a to $4 \mathrm{c}$, and a similar morphologic change is observed in the histologic images. Nuclear density increases with the imaging depth in the SECM images, and a similar trend is shown in the histologic images.

\section{Discussion}

We demonstrated the feasibility of conducting automatic, comprehensive confocal microscopy of the esophagus in vivo using SECM. Very large confocal images $\left(11 \mathrm{~cm}^{2}\right)$ of the swine esophagus from three imaging depths were obtained in about 2 minutes. Low-magnification views enabled the visualization of the gross morphology of the tissue, while high-magnification views revealed cellular features. This technology can also be modified to be used to image large portions of other gastrointestinal tract organs, including the stomach, small intestines, colon, and rectum. There were, however, areas where the technology needs to be improved further. Artifacts were present in portions of the SECM images. The first artifact was likely caused by peristalsis, where tissue motion relative to the SECM probe resulted in a zig-zag pattern in some regions ( $\bullet$ Fig. 2 a, arrowheads). A second artifact was loss of contact between the probe and the tissue ( $\bullet$ Fig. 2 a, NC). Adding a suction tube on the side of the probe increases and stabilizes tissue contact [13], which will likely reduce these two artifacts. The processing time to generate a large-area image such as $\bullet$ Fig. 2 a (file size $=1.3$ Gbytes) was around 4 minutes. This amount of processing time is acceptable for post-opera- 


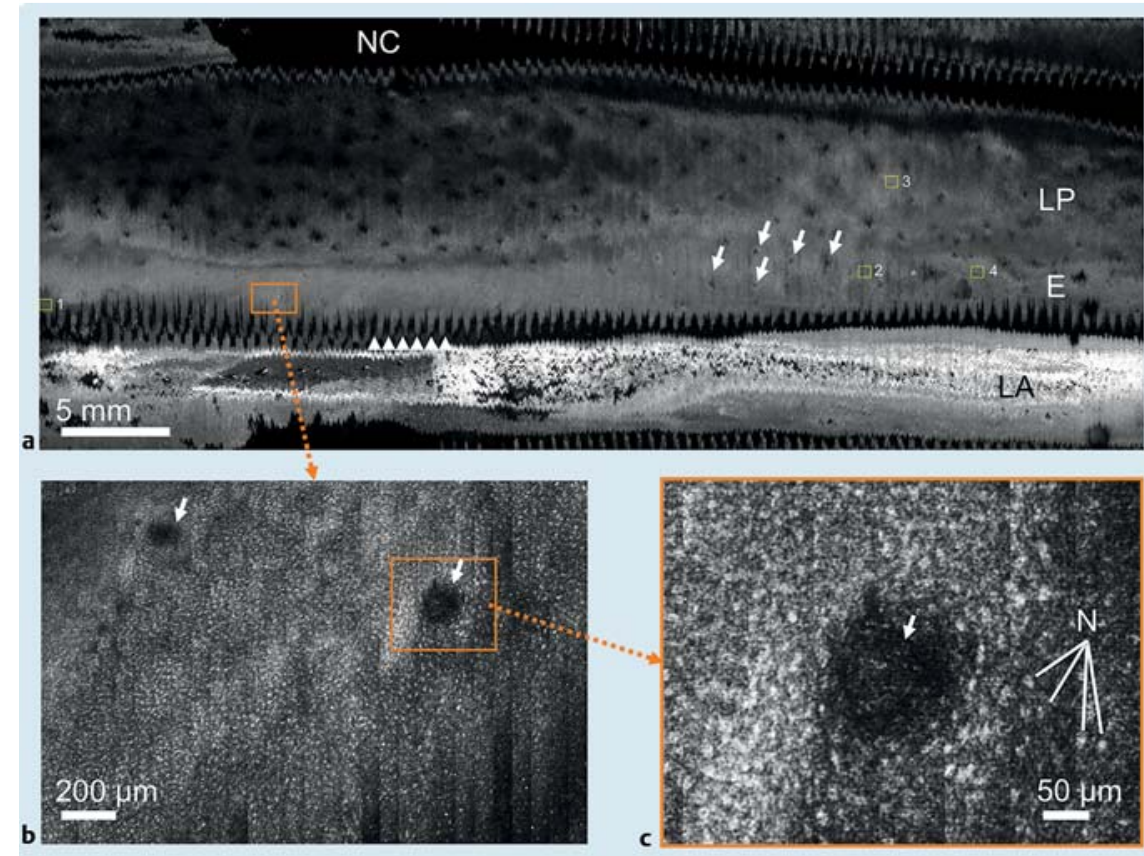

Fig. 2 SECM images of the swine esophagus in vivo. a Low-magnification image; b magnified view of Fig. $\mathbf{2}$; $\mathbf{c}$ magnified view of Fig. $\mathbf{2} \mathbf{b}$. $\mathrm{NC}$, no contact between the tissue and imaging tube; LP, lamina propria; E, epithelium; LA, reflected light from the air-liquid boundary; N, nuclei; arrows, papillae; and arrowheads, zig-zag pattern on the tissue surface.

tion image analysis. However, a shorter processing time will be needed if we want to conduct intraprocedural visualization of the entire confocal microscopy dataset. Because of the current imaging depth of $100 \mu \mathrm{m}, \mathrm{SECM}$ was capable of visualizing the epithelium and LP but was unable to be used to image deeper tissue regions such as muscularis mucosa and submucosa. Optical coherence tomography (OCT) has been successfully used to visualize architectural features of these deeper tissue regions [14]. Because conditions such as Barrett's esophagus (BE) and esophageal squamous dysplasia are primarily limited to the superficial mucosa, we expect that the cellular features visualized by SECM will provide comprehensive diagnostic information for most screening and surveillance cases.

As the next step of this research, we will utilize this probe to obtain comprehensive confocal microscopy images of the human esophagus in vivo. When imaging the human esophagus, we will use a lower concentration of acetic acid (1-3\%) than the concentration used for the swine esophagus (6\%), since our previous study of imaging human esophageal biopsy tissues showed that $0.6 \%$ acetic acid provides sufficient nuclear contrast in human tissues [8]. The transparent imaging tube will be disposed after each imaging session, while the internal SECM probe optics will be used for multiple imaging sessions. The costs of the imaging probe and disposable transparent imaging tube will likely be comparable to the costs of other imaging devices that are used in conjunction with endoscopy.

SECM images could enable targeted biopsy based on microscopic tissue assessment of the entire distal esophagus. During the imaging procedure, SECM images will be displayed in real time while the SECM probe automatically scans a long segment of the esophagus. An endoscopist or pathologist will review the SECM images and will determine regions that need further examination by histopathology. In conjunction with the laser marking functionality [15], the SECM probe can generate visible marks around the identified tissue regions and guide the endoscopist to biopsy tissues at these regions. The targeted biopsy enabled by SECM imaging could be helpful for improving the sampling yield of biopsy procedures such as those performed for Barrett's surveillance. For endoscopists or pathologists to be able to read
SECM images, we will develop and validate diagnostic criteria for SECM interpretation. Operators will undergo training sessions to gain expertise in reviewing and interpreting SECM videos and images.

Several challenges, however, are expected during SECM imaging of the human esophagus in vivo, including following four. First, certain shapes of the distal esophagus might pose difficulties for SECM imaging, including esophageal stricture or tortuosities. For these cases, it may be difficult to pass the probe as it would be for conventional endoscopy. These geometrical distortions can also bend the probe, which can bind the driveshaft, causing rotational artifacts. If the patient has an esophagus that is larger than normal, the SECM probe may not come into contact with a large portion of the esophagus. This situation may be mitigated by obtaining one pullback SECM scan and then repositioning the probe to acquire images from another region of the esophagus. Second, luminal contents, which are occasionally present in patients with gastroesophageal reflux disease (GERD) or BE, might make the SECM imaging difficult. The luminal contents could enter the space between the SECM probe and tissue, which can attenuate the light and change the focal plane so that it is no longer within the tissue itself. To mitigate this factor, we ensure that luminal contents are cleared during the initial endoscopic examination. As a result, we do not expect that luminal debris will interfere with SECM imaging. Third, the current SECM endoscopic probe always requires video endoscopy to introduce a guide wire. Conducting SECM imaging in conjunction with video endoscopy is beneficial since the esophagus can be cleaned during the endoscopy procedure, acetic acid can be applied in a controlled manner, and the location of the gastroesophageal junction (GEJ) can be determined, which guides SECM probe placement. Fourth, due to its current diameter of $7 \mathrm{~mm}$, the SECM probe cannot be introduced through the accessary port of a standard video endoscope. Compared to the current over-the-guide wire introduction of the SECM probe, through-the-scope SECM could facilitate good spatial registration between SECM images and endoscopic observation. To utilize the SECM probe through the endoscopic accessory port, the diameter of the probe will need to be reduced. 

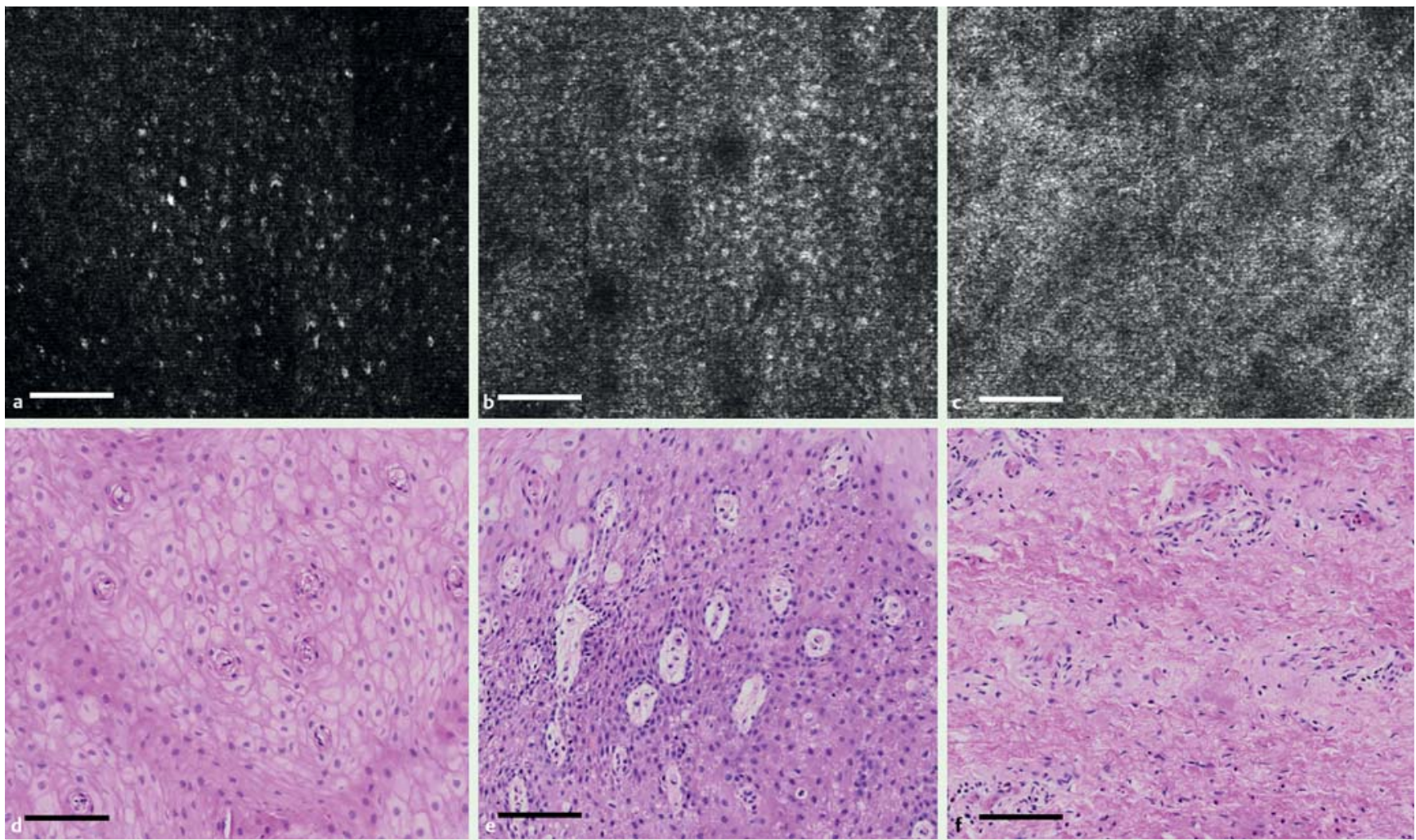

Fig. 3 High-magnification SECM images (a, b, $\mathbf{c})$ and representative, nearby en face histologic images $(\mathbf{d}, \mathbf{e}, \mathbf{f})$ of swine esophagus. SECM images were taken from the regions marked by the boxes 1, 2, and 3 in $\mathbf{~ F i g . ~} \mathbf{2}$ a. a and $\mathbf{d}$ Stratified squamous epithelium; $\mathbf{b}$ and $\mathbf{e}$ basal cell layer; $\mathbf{c}$ and $\mathbf{f}$ lamina propria (scale bar $=100 \mu \mathrm{m})$
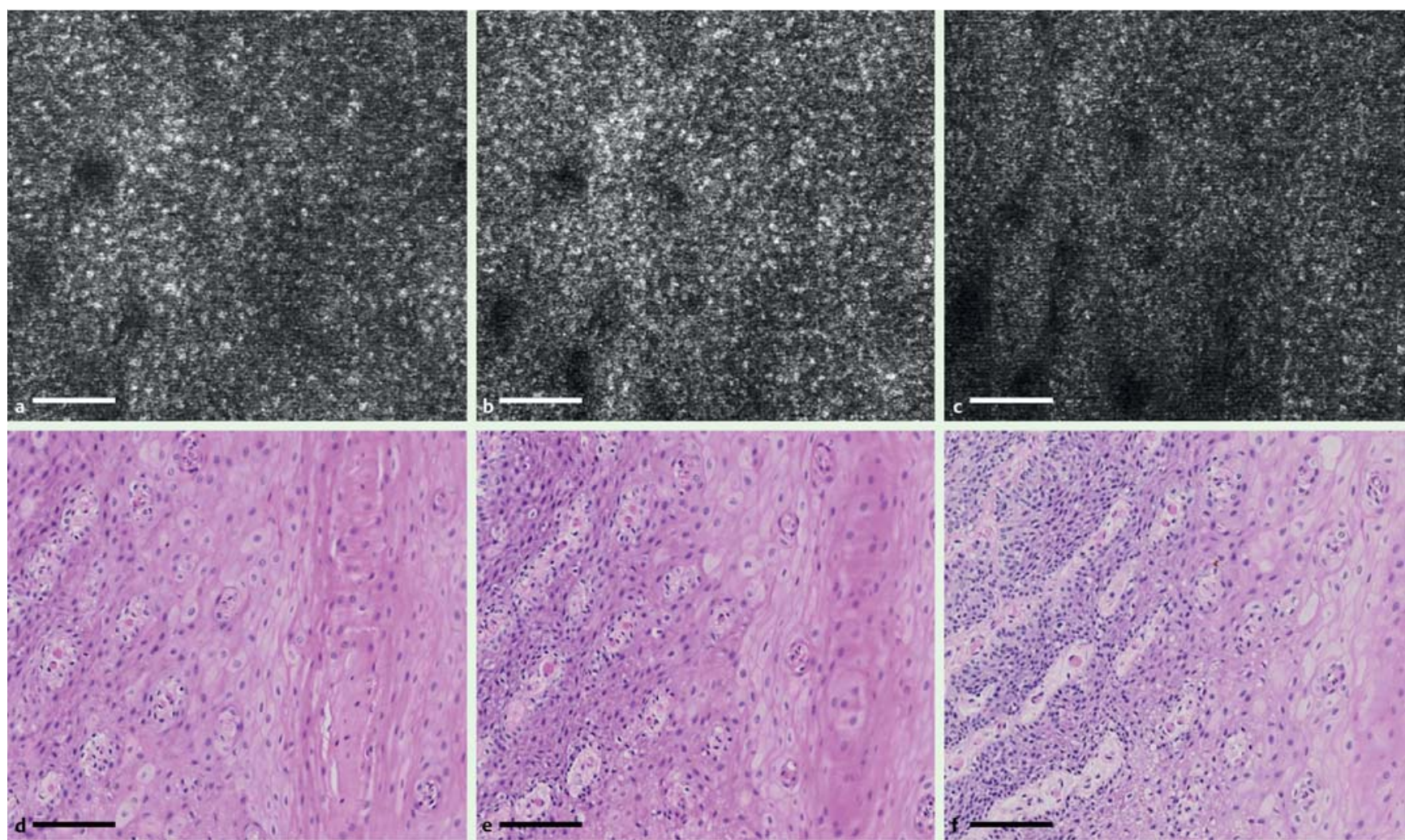

Fig.4 SECM and representative, nearby en face histologic images taken at the same respective transverse locations but at different imaging depths. The imaging depth changes from superficial (a and $\mathbf{d}$ ) to deep (c and $\mathbf{f})$, with a depth difference of $14 \mu \mathrm{m}$ between images. The SECM images were taken from the region marked by box 4 in $\$$ Fig. 2 a (scale bar $=100 \mu \mathrm{m})$. 


\section{Abbreviations}

$\nabla$

BE Barrett's esophagus

CLE Confocal laser endomicroscopy

GEJ gastroesophageal junction

GERD gastroesophageal reflux disease

LP Lamina propria

OCT Optical coherence tomography

SECM Spectrally-encoded confocal microscopy

Competing interests: G. J. Tearney received sponsored research from Nine Point Medical. M. Shishkov, K. Woods and G. J. Tearney consult for Nine Point Medical. Massachusetts General Hospital has a licensing arrangement with Nine Point Medical. D. Kang, S. C. Schlachter, R. W. Carruth, N. Tabatabaei, M. Shishkov, J. S. Sauk, N. S. Nishioka, G. J. Tearney have the rights to receive royalty payments as part of this licensing arrangement.

Institutions

${ }^{1}$ Massachusetts General Hospital - Wellman Center for Photomedicine, Boston, MA

2 The University of Tokyo -School of Engineering, Tokyo, Japan

${ }^{3}$ Massachusetts General Hospital - Department of Gastroenterology, Boston, MA

${ }^{4}$ Harvard-MIT Division of Health Sciences and Technology, Cambridge, MA

${ }^{5}$ Massachusetts General Hospital -Department of Pathology, Boston, MA

\section{Acknowledgment}

The authors thank William Puricelli, RN for his help during the animal study. This research has been sponsored by Nine Point Medical, Cambridge, MA. S. C. S is currently with Nine Point Medical. K. W. is currently with Emory University Hospital, Atlanta, GA. J. L. is currently with Tufts Medical Center, Boston, MA.

\section{References}

1 Kiesslich R, Gossner L, Goetz M et al. In vivo histology of Barrett's esophagus and associated neoplasia by confocal laser endomicroscopy. Clin Gastroenterol Hepatol 2006; 4: 979-987

2 Kitabatake S, Niwa Y, Miyahara $R$ et al. Confocal endomicroscopy for the diagnosis of gastric cancer in vivo. Endoscopy 2006; 38: $1110-$ 1114

3 Buchner AM, Shahid MW, Heckman MG et al. Comparison of ProbeBased Confocal Laser Endomicroscopy With Virtual Chromoendoscopy for Classification of Colon Polyps. Gastroenterology 2010; 138: 834 842

4 Meining A, Chen YK, Pleskow D et al. Direct visualization of indeterminate pancreaticobiliary strictures with probe-based confocal laser endomicroscopy: a multicenter experience. Gastrointestinal endoscopy 2011; 74: 961 - 968

5 Konda VJ, Aslanian HR, Wallace MB et al. First assessment of needlebased confocal laser endomicroscopy during EUS-FNA procedures of the pancreas (with videos). Gastrointestinal endoscopy 2011; 74: $1049-1060$

6 Becker $V$, Vercauteren $T$, von Weyhern $\mathrm{CH}$ et al. High-resolution miniprobe-based confocal microscopy in combination with video mosaicing (with video). Gastrointest Endosc 2007; 66: 1001 - 1007

7 Tearney GJ, Webb RH, Bouma BE. Spectrally encoded confocal microscopy. Opt Lett 1998; 23: $1152-1154$

8 Kang D, Suter MJ, Boudoux C et al. Comprehensive imaging of gastroesophageal biopsy samples by spectrally encoded confocal microscopy. Gastrointest Endosc 2010; 71: 35-43

9 Yoo H, Kang D, Katz AJ et al. Reflectance confocal microscopy for the diagnosis of eosinophilic esophagitis: a pilot study conducted on biopsy specimens. Gastrointest Endosc 2011; 74: 992 - 1000

10 Kang D, Carruth RW, Kim M et al. Endoscopic probe optics for spectrally encoded confocal microscopy. Biomed Opt Express 2013; 4: 19251936

11 Kang $D$, Yoo $H$, Jillella $P$ et al. Comprehensive volumetric confocal microscopy with adaptive focusing. Biomed Opt Express 2011; 2: $1412-1422$

12 Carlson K, Pavlova I, Collier T et al. Confocal microscopy: Imaging cervical precancerous lesions. Gynecologic oncology 2005; 99: 84-88

13 Zhang Y-M, Bergman JJ, Weusten B et al. Endoscopy Innovation Forum: Radiofrequency Ablation for Early Esophageal Squamous Cell Neoplasia. Endoscopy 2010; 42: 327-333

14 Suter MJ, Vakoc BJ, Yachimski PS et al. Comprehensive microscopy of the esophagus in human patients with optical frequency domain imaging. Gastrointest Endosc 2008; 68: 745 - 753

15 Suter MJ, Jillella PA, Vakoc BJ et al. Image-guided biopsy in the esophagus through comprehensive optical frequency domain imaging and laser marking: a study in living swine. Gastrointestinal Endoscopy 2010; 71: 346-353

16 Thiberville L, Salaün M, Lachkar S et al. Confocal fluorescence endomicroscopy of the human airways. Proceedings of the American Thoracic Society 2009; 6: 444-449

17 De Palma GD, Staibano S, Siciliano $S$ et al. In vivo characterisation of superficial colorectal neoplastic lesions with high-resolution probebased confocal laser endomicroscopy in combination with video-mosaicing: A feasibility study to enhance routine endoscopy. Digestive and Liver Disease 2010; 42: 791 - 797 